\title{
Recording transcriptional activity
}

\author{
Acquisition of RNA into CRISPR arrays allows the recording of \\ transcriptional dynamics in cells.
}

O ne core interest of Randy Platt at the ETH in Basel is understanding the molecular underpinnings of development, but he finds the principle behind current approaches limiting: "You draw these schematics of one cell differentiating into two cells. You draw an arrow, but the arrow is really a black box," he says.

The arrows represent dynamic changes in the transcriptome, but how well these are understood depends on how many time points along their path are gathered. Platt wanted to get away from sampling and instead record the dynamics of transcriptional changes directly in the cell. The problem was not the lack of an idea for how to do this-Platt envisioned a recording device based on the CRISPR system's ability to acquire new spacer sequences into the CRISPR locus; the problem was that known CRISPR systems acquire DNA only.

In 2016, Andrew Fire's group at Stanford University made a breakthrough that showed spacer acquisition from RNA. The team discovered a bacterial species that used a Cas 1 protein, fused to a reverse transcriptase, complexed with Cas2 to form an integration complex and add spacers derived from DNA or RNA into the CRISPR locus (Silas et al., 2016). Unfortunately, this RT-Cas1 complex did not retain its function in Escherichia coli.

Florian Schmidt and Mariia Cherepkova, graduate students in the Platt lab, started with a screen to find orthologous RT-Cas1 and Cas2 systems from diverse phyla in the hope that they would find one that worked across different species. Spacer acquisition is a rare event, and the researchers needed a sensitive screen for it. They developed SENECA, an approach that selectively amplified only the CRISPR arrays with new spacers. Their screen yielded several candidates, but RT-Cas1-Cas2 from Fusicatenibacter saccharivorans, when overexpressed in E. coli together with the CRISPR array that captures the spacers, showed activity 1,000-fold above that of the others. It could capture RNA from an invading phage.

The team named this spacer capture approach Record-seq and showed that it could quantitatively record a history of the transcriptional activity of both reporter genes and endogenous $E$. coli genes altered in response to oxidative and acid stress. They also were able to record dose-dependent and transient expression in response to bacteriostatic herbicide exposure.

So far the researchers have comprehensively characterized only one RT-Cas1-Cas2 complex, but Platt describes the other orthologs as "a treasure trove of potentially new transcriptional recorders." They are currently working on getting Record-seq to work in eukaryotic cells. "It would be interesting to use this in mammalian cells and understand how expression changes over time and through development," says Platt.

In their initial work the team focused on the expression of a single gene per experiment, but they found many CRISPR arrays with spacers from different genes, and they are looking into how to best use this information to reconstruct transcriptional dynamics.

Platt is quick to stress that Record-seq and SENECA are not just approaches to profile transcriptional dynamics. He also sees them as useful for learning more about basic CRISPR biology and understanding more about spacer acquisition. He explains that the current understanding of CRISPR adaptation is derived from the investigation of only a few CRISPR system subtypes. He adds, "We hope that this can be used by the community to look at diverse types of CRISPR systems that have historically been intractable."

Since the paper was published, he has received many requests for reagents and suggestions for future work. "The ideas that people are coming up with in order to implement this are fascinating," he says. "I am excited to see where this technology will take us."

Nicole Rusk

Published online: 30 November 2018 https://doi.org/10.1038/s41592-018-0242-5

Research papers

Silas, S. et al. Direct CRISPR spacer acquisition from RNA by a natural reverse transcriptase-Casl fusion protein. Science 351, aad4234 (2016).

Schmidt, F. et al. Transcriptional recording by CRISPR spacer acquisition from RNA. Nature 562, 380-385 (2018).

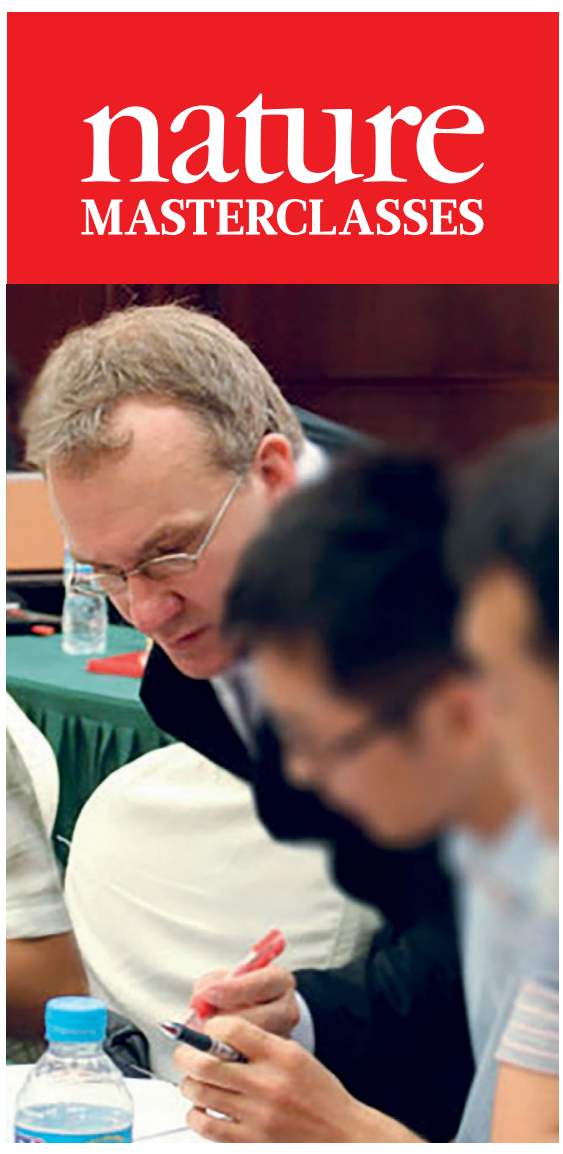

Workshops in

Scientific Writing

and Publishing

Delivered by Nature Research

journal editors and hosted

at institutions worldwide,

researchers learn how to

turn great science into great

papers.

Find out more at

masterclasses.nature.com

W masterclasses.nature.com

in Follow us on Linkedln 